\title{
Fabrication of Tm2O3/A12O3-silica preform by improved MCVD-chelate delivery system
}

\begin{abstract}
The fabrication of Tm/Al-doped silica preforms by an improved MCVD method with metal chelate precursors is discussed. Two fabrication techniques are employed, namely; simultaneous soot-dopant deposition (or standard MCVD) and stepwise soot-dopant deposition. The preforms are characterized by refractive index profiler and EPMA. The results show that the stepwise soot-dopant technique has a higher incorporation of $\mathrm{Al} 2 \mathrm{O} 3$ and $\mathrm{Tm} 2 \mathrm{O} 3$ as compared to the simultaneous soot-dopant method. This is due to the drawbacks of our chelate delivery system such as the temperature gradient and flow design. For the stepwise technique, the measured index difference of the preform is 0.006 with $0.8 \mathrm{wt} \%$ (maximum) Tm incorporated in the core.
\end{abstract}

Keyword: EPMA; MCVD; Metal chelate; Rare earth doped silica; Refractive index 